\title{
Interdisciplinarity as Departure and Return: Methodological Boundary Crossing in the Ancient Near East
}

\section{Isaac M. Alderman', Shane M. Thompson ${ }^{2}$, and Eric M. Trinka ${ }^{3}$}

At the time this inaugural issue of Avar: A Journal of Life and Society in the Ancient Near East heads to press, we are keenly aware of the fact that the number of new journals in the fields of Biblical Studies, Egyptology, and broader Ancient Near Eastern Studies have proliferated in the last decade. Yet, we hope to demonstrate to our readers that Avar fills an important lacuna in the academic study of the ancient past. The title of the journal, Avar, centers interdisciplinarity as the primary framework for illuminating life and society in the ancient Near East. In what follows, we will introduce our vision for such interdisciplinarity.

Any scholar hoping to account for life and society in the ancient Near East and eastern Mediterranean must tackle a complex overlap of regions, time periods, languages, and anthropological subfields that generally exceeds one's specific locational and chronological expertise. Scholars of the ancient world have long drawn from disparate disciplines in their analyses of material cultural and textual evidence. Such work has been posited on assumptions that past and

\footnotetext{
${ }^{1}$ Isaac M. Alderman, Baruch College, United States. E-mail: isaac.alderman@gmail.com.

${ }^{2}$ Shane M. Thompson, North Carolina Wesleyan College, United States.

E-mail: sthompson@ncwc.edu.

${ }^{3}$ Eric M. Trinka, James Madison University, United States. E-mail: trinkaem@jmu.edu.
} 
present human experiences maintain consistency across time and space, even as political, social, and cultural particularities differ.

These scholars of the ancient Near East have frequently envisioned interdisciplinary work as the crossing of chronological, regional, and methodological boundaries between traditionally siloed subdisciplines (i.e., Assyriology, Biblical Studies, Egyptology, Hittitology, etc.). Combining philology with archaeology or other anthropological approaches has become normative. Likewise, merging multiple geographic regions of study has reformulated traditionally observed boundaries so that regions once considered beyond the ancient Near East have been brought within the common investigative purview. These popular approaches broadly promote comparative studies of language, texts, religion, households, trade, politics, and material culture across regions. While comparative endeavors are useful and have produced countless important findings, such work does not wholly satisfy the criteria for interdisciplinarity cultivated by interdisciplinary studies as a field unto itself. To be interdisciplinary, scholarship must embrace approaches that are not traditionally in conversation with the datasets or methodologies already present within a given field. Such work is informed by areas of study beyond our shared chronological and geographical scopes that can illuminate our evidence in fresh ways.

From our earliest conversations as an editorial team, we have conceptualized Avar's mission in geographic terms. The journal's moniker in Hebrew ( $\underline{b}$ r עבר) denotes both human transience and border crossing. Its preceding Akkadian cognate (ebèru/ebāru) refers to similar acts of crossing over land features or borders. Historically, the lands of Mesopotamia were called 'abr nabarin "the land across the rivers" in Aramaic. In keeping with the metaphor of movement, one must venture beyond the comforts of their home disciplinary territory with the intention of returning with scholarly tools or novel approaches that will augment earlier methodologies and hopefully produce new insights. Avar promotes interdisciplinarity as an act of crossing characterized by departure and return. We envision scholars not only moving across disciplinary boundaries but, through their 
movement, we also anticipate they will reconceptualize the boundaries that currently organize their sub-disciplines.

We are not the first to conceive of interdisciplinarity in geographical terms. The phrase "boundary work" has been applied by scholars of interdisciplinarity since the early 1980s to demonstrate that all work within and between disciplines involves the setting, moving, or rearranging of various boundaries that define the limits of the discussion at hand. ${ }^{4}$ More than a decade before that, in 1970, a committee on teaching and research named the concerns of interdisciplinarity. ${ }^{5}$ Working in the context of this earlier scholarship, Julie Thompson Klein wrote that interdisciplinarity is "when integration and interaction become proactive," and is marked by the verbs "Interacting, Integrating, Focusing, Blending, Linking." "While many scholars are drawn to interdisciplinary research because of what might be considered simple curiosity, the setting or rearranging of these boundaries necessarily involves choices that are political or in the context of relationships of power. These dynamics have been the object of research in themselves now for more than five decades and it is important for those in our fields who want to do interdisciplinary work to recognize and utilize this research.

Klein created a taxonomy of three rounded categories of interdisciplinarity. The first, methodological interdisciplinarity seeks to utilize multiple disciplines in order to improve the accuracy of results. Very often here we find methodologies from one discipline used to supplement weaknesses found in another. Another instance would be using methods from another discipline to analyze or critique your

\footnotetext{
${ }^{4}$ Klein adopts this language from Gieryn. Julie Thompson Klein, "Typologies of Interdisciplinarity: The Boundary Work of Definition," in The Oxford Handbook of Interdisciplinarity (Oxford: Oxford University Press, 2017), 21-34; Thomas P. Gieryn, "Boundary-work and the Demarcation of Science from Non-science: Strains and Interests in Professional Ideologies of Scientists," American Sociological Review 48 (1983): 781-95.

${ }^{5}$ Leo Apostel, ed., Interdisciplinarity: Problems of Teaching and Research in Universities (Paris: Organization for Economic Co- operation and Development, 1972).

6 Julie Thompson Klein, Crossing Boundaries: Knowledge, Disciplinarities, and Interdisciplinarities (Charlottesville: University Press of Virginia, 1996), 6.

${ }^{7}$ Klein, "Typologies," 22.
} 


\section{Interdisciplinarity as Departure and Return}

own discipline. ${ }^{8}$ The goal of theoretical interdisciplinarity is to create conceptual frameworks, drawing from across disciplines, to analyze problems, or develop models and analogies. Often, this involves using the insights and concepts from one discipline to contribute to the problems and theories of another, which can result in new conceptual categories or the modification of one's own discipline. ${ }^{9}$ The last category, bridge-building/restructuring, involves separate, but related, moving of disciplinary boundaries. In bridge-building, distinct and whole disciplines are united through a new methodology or approach that draws them together. In the insurance of restructuring, aspects of more than one discipline are disconnected from their original context and united to form a new disciplinary category. ${ }^{10}$ One purpose of sharing our view here is to be clear about what is and is not interdisciplinary, since many publications often claim to be interdisciplinary when they are not. The goal is not to be pedantic, but to be clear about purposes and methodology. These various differences have been so well outlined by scholars of interdisciplinarity that there is no need for us to be muddled in our terminology. The intention of interdisciplinarity is not merely to bring external evidence or epistemologies to bear on our present fields of study but to allow findings in our present scholarly domains to enrich those fields from which we draw new methods and ways of seeing.

In our general call for submissions, we welcome papers that cross disciplinary boundaries by explicitly adopting, adapting, or integrating theories and methodologies from within the traditional fields of ancient studies with socio-anthropological and scientific disciplines. Interaction with other disciplines should be clear, using shared language, theories, and ideas already established, and enhancing the scope of the conversation regarding such shared questions. Such contributions will take the form of single author papers that demonstrate immersive theoretical or methodological

\footnotetext{
${ }^{8}$ Klein, "Typologies," 24-25.

${ }^{9}$ Klein, "Typologies," 25-26.

${ }^{10}$ Klein, "Typologies," 26.
} 
approaches and illuminate historical evidence and contexts in novel ways. They may also include multi- author papers co-written by specialists from different disciplines.

Part of the challenge of interdisciplinary work is that it pushes against our own cognitive structures which form disciplinary boundaries as a means of retaining and organizing information in a process called "cognitive chunking." These boundaries assist in memory, and shape experience and interpretation. ${ }^{11}$ Our scholarship is inherently informed by human patterns of thought which connect cognitive structures. For example, a scholar's use of terminologies such as "imperialism," "culture," or "migration" do not reflect terms found in ancient Near Eastern languages and mindsets, but, instead, concepts present within the worldview of modern scholars. As modern scholars, we inherently make these connections between the ancient and modern worlds through our own lenses, yet often leave theoretical discussions of these concepts behind due to their pervasive nature within our own society. We are not calling for a dissolution of disciplinary boundaries but for the creation of networks of intellectual hospitality and mutuality that result in cross pollination, and therefore, demonstrate the necessity of ancient history, archaeology, and textual study in the present academic landscape. In fact, we suggest that such an approach will also encourage scholars of other disciplines to engage with work on the ancient Near East within their own scholarship.

Some scholars are already doing the type of interdisciplinary work we hope to publish in Avar. In recent years, we have witnessed an increase of approaches that incorporate anthropological, sociological, and psychological data and models along with methods and findings from hard sciences. The merger of these methodological worlds has often come by way of archaeology with its anthropological heritage. More recently, reconstructions of the ancient past have been expanded to include robust studies of social

\footnotetext{
11 Iva K. Brunec, Morris Moscovitch, and Morgan D. Barense, "Boundaries Shape Cognitive Representations of Spaces and Events," Trends in Cognitive Sciences 22 (2018): 637-650.
} 
phenomena and investigations of the human condition. We affirm these investigative trajectories and wish to further hone the practices of interdisciplinarity for the study of life and society in the ancient Near East. In doing so we aim to publish content that pushes beyond the boundaries of traditional multidisciplinary or cross-disciplinary study in the humanities.

Our vision for the future of interdisciplinary work on the ancient Near East stands behind the topic of our first issue: deviance. In the same sense that disciplinary boundaries exist as cognitive structures, interdisciplinary work then exists as a deviation from the norm. While we encourage the bi-directional movement of crossing and return, with this first issue we hope to emphasize the initial movement away from traditional approaches. Just as we insist on a deviation from the norm leading to more interdisciplinary work in our scholarship on the ancient Near East, we now hope to highlight deviance and deviation from the norm in aspects of life from the ancient Near East.

While deviance is a modern category not expressly operating in ancient evidence, we recognize the broader patterns of normativity and deviance that attend past and present human experience. In our call for this issue, we invited papers addressing human differences characterized by deviance, practices and attitudes toward deviance, methodological approaches to studying deviance and discourses of deviance, and investigations of the relationship between deviance and criminality. We have invited authors to conceptualize deviance in manifold ways in their readings and reconstructions of the ancient past. They have brought their own conceptions of the term to their work, highlighted by interaction with scholarship on deviance from other fields.

The construction of knowledge is a collective enterprise. We are grateful to Transnational Press London for its willingness to serve as a home for Avar. Likewise, this inaugural issue would not be possible without the support and work of our advisory board, editorial board, or referees. 